Journal of Mathematics and Informatics

Vol. 20, 2021, 51-60

ISSN: 2349-0632 (P), 2349-0640 (online)

Published 20 April 2021

www.researchmathsci.org

DOI: http://dx.doi.org/10.22457/jmi.v20a06193

Journal of

Mathematics and Informatics

\title{
Based on Improved Level Set Algorithm to Enhanced Fuzzy C-Means Clustering for Controlled Knife Image Segmentation
}

\author{
Cheng Yihui $^{1}$, Zhang Guicang ${ }^{1}$, Han Genliang ${ }^{2,1}$ and Song Yuzhe ${ }^{2}$ \\ ${ }^{1}$ School of Mathematics \& Statistics, Northwest Normal University \\ Gansu Lanzhou, 730070, China. Email: 2019211694@ nwnu.edu.cn \\ ${ }^{2,1}$ Key Laboratory of Sensor and Sensor Technology of Gansu Province \\ Gansu Lanzhou, 730070, China. Email: genlhan@vip.sina.com \\ ${ }^{2,2}$ Key Laboratory of Sensor and Sensor Technology of Gansu Province \\ Gansu Lanzhou, 730070, China. Email: songyuzhe@ 126.com \\ ${ }^{*}$ Corresponding author
}

Received 10 March 2021; accepted 18 April 2021

\begin{abstract}
With the deepening of China's urbanization construction, public safety is also facing great challenges. Therefore, China has strengthened security measures for important public places, and established monitoring network and security inspection system. However, the current machine security inspection algorithm is not accurate enough for the identification of sensitive objects such as controlled knives, and it also depends on manual detection and confirmation, which leads to low work efficiency and often leads to low efficiency It is also not conducive to the prevention and control of the epidemic. This paper proposes an image segmentation method based on Improved level set to enhance fuzzy clustering. In this paper, the fuzzy clustering method and the improvement of the level set method, first of all, using morphological method image preprocessing algorithms, so as to realize the foreground and background of the area range, is advantageous for the next level set segmentation of the target outline, so as to determine the fuzzy clustering of heart, makes strengthening fuzzy clustering on its own the advantage of rapid more accurate. Through experiments, the effectiveness of the algorithm is verified, and the segmentation accuracy is higher than the traditional segmentation algorithm.
\end{abstract}

Keywords: Image segmentation; level set method; EnFCM; morphology

AMS Mathematics Subject Classification (2010): 94A08 
Cheng Yihui, Zhang Guicang, Han Genliang and Song Yuzhe

\section{Introduction}

Public security has always been an important part of national security, an important part of the government's social management and public service, and also one of the most concerned focuses of the public. Therefore, the airport, stations, docks, hospitals and other public places have strengthened security measures, set up a monitoring network and security inspection system, to a large extent, effectively guaranteed the safety of people's lives and property. However, the current machine security algorithm for guns, controlled knives and other sensitive objects recognition accuracy is not high enough, but also rely on manual detection and confirmation, resulting in work efficiency is not high enough, often resulting in personnel aggregation, which is not conducive to epidemic prevention and control. The outbreak of COVID-19 also made us realize that the importance of security monitoring can be effectively identified. Therefore, it is of practical significance and long-term value to solve this problem.

The solution of this problem mainly depends on image segmentation. Image segmentation is the key link in image processing, which plays a decisive role in subsequent image processing and image recognition. The accuracy and universality of segmentation is the key to study segmentation algorithms. At present, there are many segmentation algorithms, especially those based on various theories, such as data-driven segmentation, model-driven segmentation, physical model, random field model and semi-automatic method [1-4], etc. However, no matter which method is used, the influence of noise in the segmentation process is still not negligible. Of wavelet analysis in wide application in image processing, such as multi-scale edge detection, image recognition, image segmentation, the wavelet can be to scale the image thinning, at the same time can be separated according to the coefficient of the noise so as to realize the denoising effect, for example, through improved threshold function, make it on the real number domain to continuously, which can reduce the distortion[5-6]. The level set method in active contour model is widely used in image segmentation because of its simple numerical calculation, which mainly uses the curvature and normal vector of the curve to evolve the level set function, and can naturally realize the change of the topological structure of the object. Active contour model (ACM) [7] was proposed by the author. In recent years, it has been widely concerned by people. Level set method is one of the members of active contour model family proposed by Osher and Sethian [8]. Edge based models usually use the gradient information of local image to force the active contour to move towards the desired boundary of the target, which is particularly effective for segmentation of images with clear boundaries. Therefore, through this method, we can get more accurate target contour.

FCM (fuzzy c-means) clustering segmentation algorithm is a data clustering method based on the optimization of fuzzy objective function[9-11]. However, the classic FCM 
Based on Improved Level Set Algorithm to Enhanced Fuzzy C-Means Clustering for Controlled Knife Image Segmentation

clustering algorithm itself also has the defects of time-consuming, memory occupation and poor robustness, so this paper uses the improved enhanced fuzzy clustering segmentation method to make the algorithm more robust[12-13]. But the various details in the image will make the background have a great impact on the foreground contour extraction, resulting in errors, and the target segmentation is not accurate. These factors will reduce the accuracy of clustering, so in this paper, the level set method is combined with it, and the clustering center is determined based on the level and segmented image contour, so as to make the segmentation more accurate.

\section{Correlation theory}

\subsection{WT (Wavelet Transform)}

Denoising methods include smoothing denoising and wavelet denoising, as well as multi-method fusion denoising. For example, the median filtering method is first adopted to denoise the image, in which the weight is adaptive. Theoretically, this can also be considered to have a certain de-noising effect. However, it can be found from the actual experimental results of de-noising images that this method ignores some details while de-noising, especially the details of the edge are also smoothed during the smoothing process, as shown in the left figure of Figure 1.

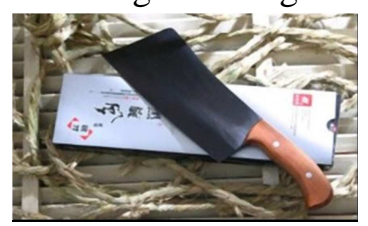

median filter

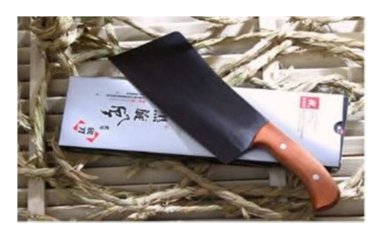

wavelet denoising

Figure 1: The median filter is compared with the denoising method in this paper

The median filtering will ignore more details than the wavelet denoising method. In this paper, the wavelet function is first used to decompose the image in two layers, and then the high frequency wavelet coefficient is processed by the threshold of two-dimensional wavelet reconstruction, and the reconstructed image is processed by the high frequency wavelet coefficient threshold again, so as to realize two times of denoising.

\subsection{EnFCM}

EnFCM (Enhanced Fuzzy C-means Clustering) is a faster image segmentation algorithm, which combines the original image with its local neighborhood by a linear weighted sum, and then performs Fuzzy mean Clustering on the gray histogram of the image. Its objective function is shown in Equation (1).

$$
J_{m}=\sum_{i=1}^{M} \sum_{j=1}^{c} r_{i} u_{j i}^{m}\left(\varepsilon_{i}-v_{j}\right)^{2}
$$


Cheng Yihui, Zhang Guicang, Han Genliang and Song Yuzhe

An average image from the original image to its local neighborhood pixels is formed by preprocessing the original image by the linear weighted summation of the pixels and their 8 neighborhood pixels. Since the grayscale value of the pixel is generally 8-bit resolution (256 grayscale levels), the encoding of the m-level grayscale value is generally much smaller than the value of the pixel size of N.As a result, execution time is significantly reduced.

$$
\begin{array}{lc}
\text { Linear weighted sum: } & \varepsilon_{\mathrm{i}}=\frac{1}{1+\mathrm{a}}\left(\mathrm{x}_{\mathrm{i}}+\frac{\mathrm{a}}{\mathrm{N}_{\mathrm{R}}} \sum_{\mathrm{j} \in \mathrm{N}_{\mathrm{i}}} \mathrm{x}_{\mathrm{j}}\right) \\
\text { Membership values: } & u_{j i}=\frac{\left(\delta_{i}-v_{j}\right)^{2 / m-1}}{\sum_{k=1}^{c}\left(\delta_{i}-v_{k}\right)^{2 / m-1}} \\
\text { center of clustering: } & v_{j}=\frac{\sum_{i=1}^{N} r_{i} u_{j i}^{m} \delta_{i}}{\sum_{i=1}^{N} r_{i} u_{j i}^{m}}
\end{array}
$$

$r_{i}$ is the number of pixels with gray value $\mathrm{i}$

\subsection{Improved level set algorithm}

Curve evolution is the motion of curve $C$ with time $t$, especially the motion of points on curve $\mathrm{C}$.

The unit normal vector $\vec{N}$ and the curvature $\mathrm{k}$ are generally used to describe the geometric features of the curve. The unit normal vector represents the evolution direction of the curve, and the curvature represents the bending degree of the curve[14]. In image segmentation, the energy on the image is used to make the plane curve move towards the target boundary.

Let $\mathrm{C}(\mathrm{p}, \mathrm{t})=(\mathrm{z}(\mathrm{p}, \mathrm{t}), \mathrm{y}(\mathrm{p}, \mathrm{t}))$ be a closed curve in $R^{2}$ that varies with time, where $p$ is the arc length parameter and $t$ is the time. Then the following partial differential equation can be used to represent the change of the curve with time:

$$
\frac{\partial \mathrm{C}}{\partial \mathrm{t}}=V(p, t)=\alpha T(p, t)+\beta N(p, t)
$$

where $\mathrm{T}$ represents the direction vector and $\mathrm{N}$ represents the unit normal vector.

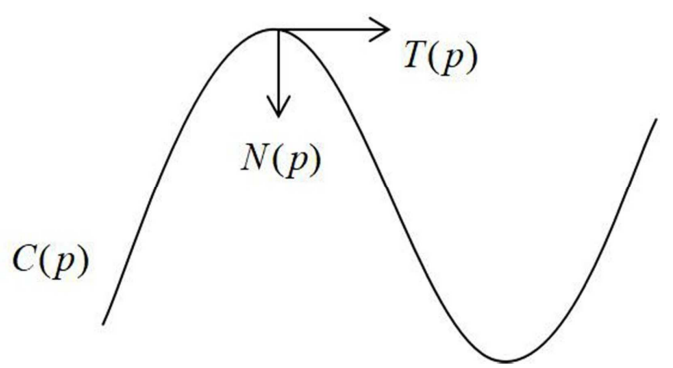

Figure 2: 
Based on Improved Level Set Algorithm to Enhanced Fuzzy C-Means Clustering for Controlled Knife Image Segmentation

Since only the velocity in the normal direction can affect the shape of the curve, while the velocity in the tangent direction will not affect the shape of the curve, the curve evolution equation can be simplified as:

$$
\frac{\partial \mathrm{C}}{\partial \mathrm{t}}=\beta N(p, t)
$$

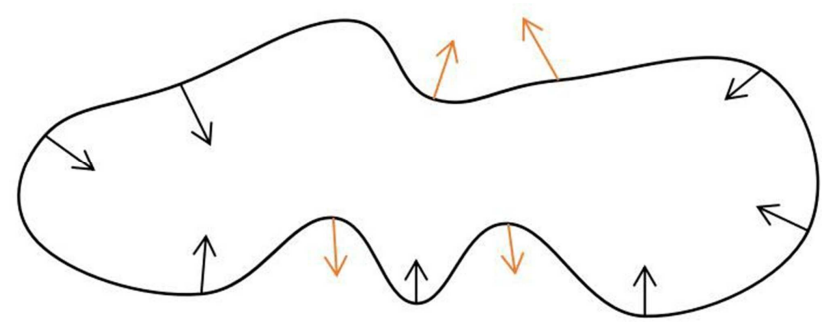

Figure 3: Curve evolution diagram

The arrow in Figure 2.1 represents the normal vector of the point on the curve. Each point on the curve moves along the direction indicated by the arrow, and the speed of movement at each point is different. Various factors work together to make the curve move toward the target boundary.

The main idea of the level set method is: first, the initial contour of a given closed, with a certain speed for the contour evolution along the normal direction inward or outward, in order to gain curve as a high-dimensional space curved surface of the zero level set, the family is called the level set function[15-16], when the contour changing topological structure, can still maintain continuity, thus ensures that this method is easy to deal with the issue of topology changes.

A curve or surface on a closed plane can be regarded as a level set function on a two-dimensional plane:

$$
\mathrm{C}=\{(\mathrm{x}, \mathrm{y}), \phi(\mathrm{x}, \mathrm{y})=\mathrm{c}\}
$$

That is flat $\phi=\mathrm{C}$ and $3 \mathrm{~d}$ surface intersecting line $\phi=\phi(\mathrm{x}, \mathrm{y})$, so we introduce the time variable $t$ get a bunch of changing with time curve $\mathrm{C}(\mathrm{t})$, $C(t)=\{(x, y), \phi(x, y, t)=0\}$, at $t$ time, of each point on the surface function of zero, the zero level set: $\{\phi=0, t\}$. Since $C(t)$ is the zero level set of the level set function $\phi(c, t)$, at the moment $\mathrm{t}$, the point $(\mathrm{x}, \mathrm{y})$ on $\mathrm{C}(\mathrm{t})$ must satisfy:

$$
\phi(\mathrm{c}(\mathrm{t}), \mathrm{t})=0
$$

Derivation of Equation (8): $\quad \frac{\partial \phi}{\partial \mathrm{t}}+\nabla \phi \cdot \frac{\partial c}{\partial t}=0$

$\nabla \phi$ is the gradient of $\phi$, it means: $\frac{\partial \phi}{\partial \mathrm{c}}=\nabla \phi=\frac{\partial \phi}{\partial \mathrm{x}}+\frac{\partial \phi}{\partial \mathrm{y}}$ 
Cheng Yihui, Zhang Guicang, Han Genliang and Song Yuzhe

Through calculation, we know that the essence of the level set method is the process of solving a time-varying partial differential equation[17-19].

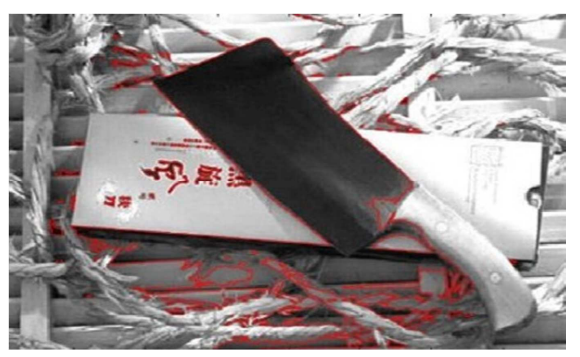

Figure 4: The image is segmented directly using the level set algorithm

We found that the contour directly segmented by level set was too complex as shown in Figure 4, which could not well separate the foreground and background, which would bring more troubles to the subsequent fuzzy clustering. Therefore, we improved it here.

Open operation can well eliminate unnecessary details in the image. After open reconstruction of the image, watershed segmentation can obviously eliminate many unnecessary contours, as shown in Figure 5.

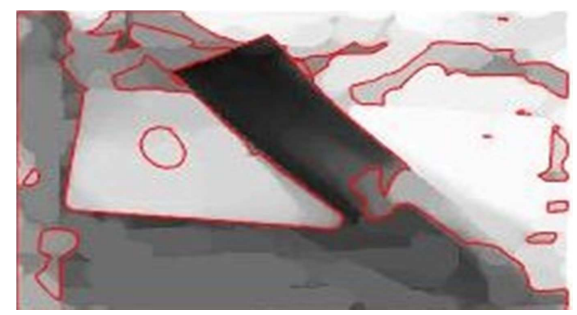

Figure 5: After open operation, the image is segmented by level set

The overall algorithm is as follows:

1.Image preprocessing using wavelet denoising;

2.The improved level set algorithm is used to obtain more accurate target contour;

3.The enhanced fuzzy clustering algorithm is adopted to segment the image under the existing contour.

\section{Analysis of experimental results}

\subsection{Experimental result}

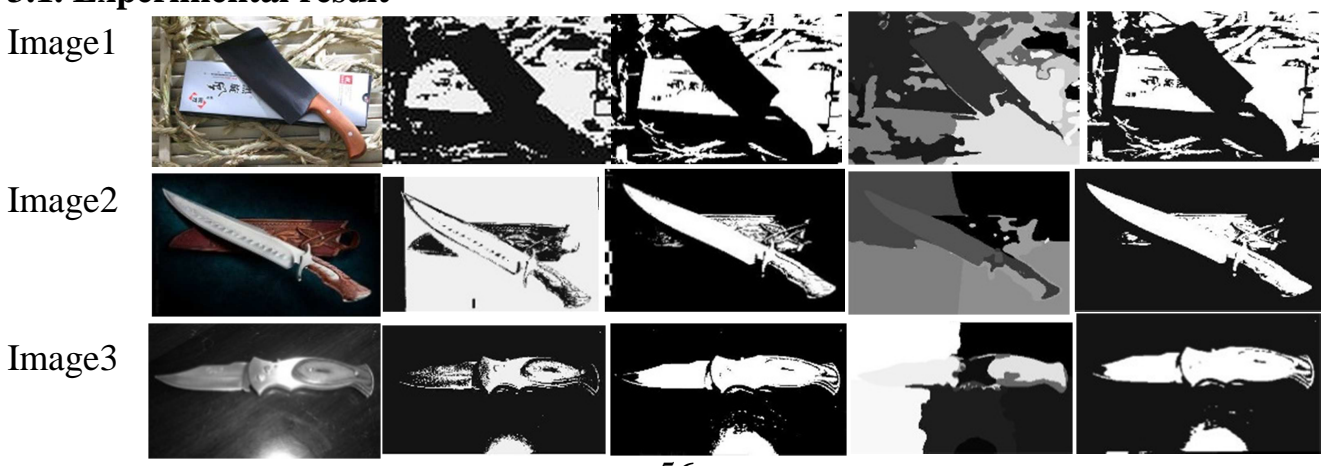


Based on Improved Level Set Algorithm to Enhanced Fuzzy C-Means Clustering for Controlled Knife Image Segmentation

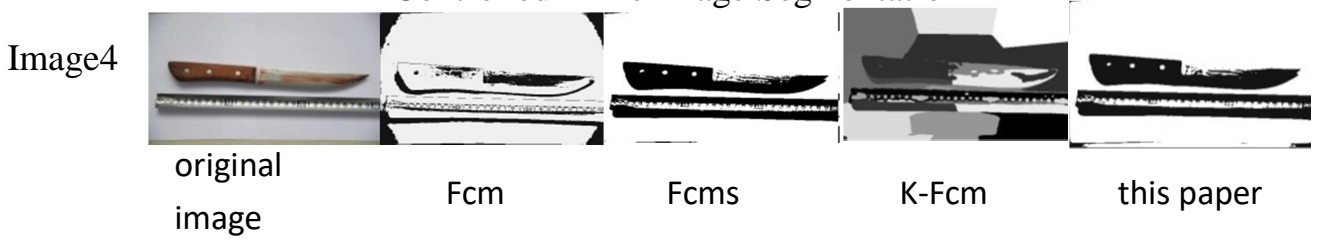

Figure 6: Experimental result

Fig. 6 shows the segmentation and comparison of five cutter images using traditional FCM algorithm, FCMS algorithm, K-means fuzzy clustering algorithm based on texture, traditional level set segmentation algorithm and the algorithm in this paper.

It can be intuitively seen that the algorithm in this paper can accurately and clearly obtain the segmentation image of the cutting tools with different backgrounds, shapes, colors and textures.

\subsection{Analysis of experimental results}

In this paper, the standard deviation, correlation coefficient and distortion degree of the segmented images obtained by the four algorithms are calculated for comparative analysis, as shown in Table 1.

Table 1: Comparison of experimental results

\begin{tabular}{|c|c|c|c|c|c|}
\hline figure & Index & $\mathrm{Fcm}$ & Fcms & $\begin{array}{c}\text { K-Fcm based } \\
\text { on texture }\end{array}$ & $\begin{array}{c}\text { The algorithm in } \\
\text { this paper }\end{array}$ \\
\hline \multirow[t]{3}{*}{1} & standard deviation & 104.3513 & 110.3193 & 82.4401 & 111.9156 \\
\hline & $\begin{array}{l}\text { coefficient } \\
\text { association }\end{array}$ & 0.6995 & 0.1855 & 0.0849 & 0.7960 \\
\hline & Distorted & 68.8518 & 104.4253 & 85.0900 & 62.5907 \\
\hline \multirow[t]{3}{*}{2} & standard deviation & 90.0438 & 90.0165 & 55.0274 & 90.5463 \\
\hline & $\begin{array}{l}\text { coefficient } \\
\text { association }\end{array}$ & -0.0663 & -0.2212 & -0.0770 & 0.1889 \\
\hline & Distorted & 174.7201 & 178.4427 & 85.5611 & 65.2251 \\
\hline \multirow[t]{3}{*}{3} & standard deviation & 72.4883 & 92.6871 & 93.3394 & 93.5349 \\
\hline & $\begin{array}{l}\text { coefficient } \\
\text { association }\end{array}$ & 0.7085 & -0.1218 & -0.1794 & 0.8071 \\
\hline & Distorted & 37.5690 & 149.3378 & 117.9121 & 37.3584 \\
\hline \multirow[t]{3}{*}{4} & standard deviation & 84.5046 & 93.7247 & 85.8340 & 102.0333 \\
\hline & $\begin{array}{l}\text { coefficient } \\
\text { association }\end{array}$ & 0.1335 & 0.4445 & 0.1706 & 0.8397 \\
\hline & Distorted & 83.2993 & 87.9040 & 85.1142 & 71.4911 \\
\hline
\end{tabular}


Cheng Yihui, Zhang Guicang, Han Genliang and Song Yuzhe

We know that the standard deviation reflects the degree of dispersion of the relative gray mean. The larger the standard deviation is, the more dispersed the gray level distribution is, and the easier it is to distinguish the foreground from the background.

The correlation coefficient of the image reflects the correlation between the two images. We compare the segmented images and the original images respectively. The larger the correlation coefficient is, the higher the similarity between the two images is.

The third index is the degree of distortion, which reflects the degree of deformation affected. The smaller the coefficient, the smaller the deformation of the image.

The results show that the segmentation algorithm has better performance and more advantages on the whole.

\section{Conclusion}

In this paper, the fuzzy clustering method and the improvement of the level set method, first of all, according to the morphology of corrosion and expansion operation for open operation, so as to realize the foreground and background of the area range, easy to split operation next level set segmentation image, and then obtain the target outline, so as to determine the fuzzy clustering of heart, made to strengthen the fuzzy clustering on its own the advantage of rapid more accurate.

The texture, color, brightness, noise, background and so on of the cutter pictures in the experiment in this paper are different, but the algorithm in this paper can still achieve more accurate and clear segmentation, which shows that this algorithm has strong robustness and strong anti-noise.

Although the purpose of this paper is to segment the prospects for the overall targets accurately, quickly to facilitate human eyes to observe whether there is a controlled knives, thus improve the security, this has been achieved in the algorithm in this paper, but we hope that we can realize the recognition of more fine, not only can filter out unwanted background details, also can keep the outlook for the necessary details.

The next step will be to study under the background of complex texture can filter out the complex background information can segment the target tool details again at the same time, such as cutting tool brand identity, special traces, and even blood, when these information preserved, we will get more information on the tool holder, to people's life and property safety can also get better security, on the other hand to the public security personnel work has brought a lot of convenient.

Acknowledgement. This work is supported by the National Natural Science Fund (NO.61861040), Applied Research and Development of Gansu Academy of Sciences(NO.2018JK-02), Key research and development plan of Gansu province(NO.20YF8GA125), Science and Technology Supported Project of Gansu 
Based on Improved Level Set Algorithm to Enhanced Fuzzy C-Means Clustering for Controlled Knife Image Segmentation

Province (NO.17YF1FA119) and Lanzhou Science and the Technology Planning Project (No : 2018-4-35).

Also, the authors are thankful to the anonymous referees for their valuable comments

\section{REFERENCES}

1. Z.C.Wu, Interactive image segmentation by maximal similarity based region merging, Pattern Recognition, 43(2) (2010) 445-456.

2. M.P.Kumar, P.Torr and A.Zisserman, OBJCUT: Efficient segmentation using top-down and bottom-up cues, IEEE Trans Pattern Anal Mach Intell, 32(3) (2010) 530-545.

3. L.Grady, Random walks for image segmentation, IEEE Transactions on Pattern Analysis \& Machine Intelligence, 28 (2006) 1768-1783.

4. D.S.Hochbaum, Polynomial time algorithms for ratio regions and a variant of normalized cut, IEEE Transactions on Pattern Analysis and Machine Intelligence, 32(5) (2010) 889-898.

5. C.J.Yong, A study on translation-invariant wavelet de-noising with multi-thresholding function, The Journal of the Acoustical Society of Korea, 25(7) (2006) 333-338.

6. R.Adelino, F.da Silva, Wavelet denoising with evolutionary algorithms, Digital Signal Processing, 15(4) (2004) 382-399.

7. M.Kass, A.Witkin and D.Terzopoulos, Snakes: active contour models, Internantional Journal of Computer Vision, 1(4) (1988) 321-331.

8. S.Osher and J.A.Sethian, Fronts propagating with curvature-dependent speed: algorithms based on Hamilton-Jacobi formulations, Computational Physics, 79(1) (1988) 12-49.

9. H.P.Mao, Y.C.Zhang and B.Hu, Segmentation of crop disease leaf images using fuzzy C-means clustering algorithm, Society of Agricultural Engineering, 24(9) (2008) 136-140 (in Chinese)

10. X.Xie, C.Wang, A.Zhang and X. Meng, A robust level set method based on local statistical information for noisy image segmentation, Optik - International Journal for Light and Electron Optics, 125(9) (2014) 2199-2204.

11. T.Lei, X.Jia, Y.Zhang, S.Liu, H.Meng and A. K. Nandi, Superpixel-based fast fuzzy C-means clustering for color image segmentation, Fuzzy Systems, IEEE Transactions on, 27( 9) (2018) 1753-1766.

12. T.Lei, X.Jia, Y.Zhang, et al. Significantly fast and robust fuzzy c-means clustering algorithm based on morphological reconstruction and membership filtering, IEEE Transactions on Fuzzy Systems, 26(5) (2018) 3027-3041. 
Cheng Yihui, Zhang Guicang, Han Genliang and Song Yuzhe

13. L.J.Zuo, C.Y.Luo and Y.X.Zuo, Paralleled segmentation cluster algorithm based on En FCM for large-scale image, Microcomputer \& Its Applications, 34(15) (2015) 55-58.

14. Y.Lei, J.Shi and J.Wu, Region-driven distance regularized level set evolution for change detectionin remote sensing images, Multimedia Tools \& Applications, 76(23) (2017) 1-16.

15. Y.Q.Zhao, X.H.Wang, X.F.Wang et al, Retinal vessels segmentation based on level set and region growing, Pattern Recognition, 47(7) (2014) 2437-2446.

16. K.H.Cha, L.Hadjiiski, R.K.Samala et al, Urinary bladder segmentation in CT urography using deep-learning convolutional neural network and level sets, Medical Physicists, 43(4) (2016) 1882.

17. M.S.Abdol and S.K.Panchal, Some new uniqueness results of solutions to nonlinear fractional integro-differential equations, Annals of Pure and Applied Mathematics, 16(2) (2018) 345-352.

18. M.Arunkumar, P.Agilan and S.Ramamoorthy, Solution and generalized ulam-hyers stability of a ndimensional additive functional equation in banach space and banach algebra: direct and fixed point methods, Annals of Pure and Applied Mathematics, 15(1) (2017) 25-40.

19. J.T.Ding and B.Z.Guo, Blow-up and global existence for nonlinear parabolic equations with Neumann boundary conditions, Computers \& Mathematics with Applications, 60 (2010) 670-679. 